\title{
Study on the binding interaction between perfluoroalkyl acids and DNA
}

\author{
Jie Cao • Yin Wei • Yan Cheng
}

Received: 15 December 2012 / Accepted: 22 April 2013 /Published online: 5 May 2013

(C) Springer-Verlag Berlin Heidelberg 2013

\begin{abstract}
Perfluoroalkyl acids (PFAAs) are carcinogens, and elucidating their DNA binding properties is crucial for understanding PFAA genotoxicity. We have investigated the binding mode and affinity of five PFAAs to seven DNA molecules using fluorescence displacement and molecular docking analysis. DNA conformational changes upon PFAA binding were also examined by circular dichroism (CD). The data revealed that DNA intercalation was the dominant interaction mode of the PFAAs; however, these molecules also bound to grooves. The dissociation constants for the PFAAs ranged between 0.11 and $1,217.14 \mu \mathrm{M}$, and between 3.46 and $2,141.21 \mu \mathrm{M}$ for DNA intercalation and groove binding, respectively. PFAAs that contain longer carbon chains had stronger DNA intercalation affinities. Binding to DNA was stronger for perfluoroalkyl sulfonates than for perfluorcarboxyl acids that contain the same number of
\end{abstract}

Responsible editor: Henner Hollert

Jie Cao and Yin Wei contributed equally to this work.

Electronic supplementary material The online version of this article (doi:10.1007/s11356-013-1760-4) contains supplementary material, which is available to authorized users.

\section{J. Cao}

AQSIQ Key Laboratory of Drug Detection, Fujian International

Travel Healthcare Center, Fujian Entry-Exit Inspection

and Quarantine Bureau of P.R.C., Fujian 350001, China

\section{Y. Wei $(\bowtie)$}

State Key Laboratory of Environmental Chemistry

and Ecotoxicology, Research Center for Eco-environmental

Sciences, Chinese Academy of Sciences,

P.O. Box 2871, Beijing 100085, China

e-mail: weiyin@rcees.ac.cn

\section{Y. Cheng}

Chinese Academy of Inspection \& Quarantine,

No. 3A, North Gaobeidian Road,

Beijing 100123, China carbons. This observation is postulated to arise from the presence of more fluorine and oxygen atoms in perfluoroalkyl sulfonates acting as hydrogen bond donors that facilitate stronger DNA intercalation. The binding of the PFAAs to DNA showed some CT-DNA sequence selectivity. Molecular docking analysis confirmed the DNA binding mode and affinities of the PFAAs. CD analysis revealed that the PFAAs weakened DNA base stacking and loosened DNA helicity. The present study has improved our understanding of the formation of PFAA-DNA adducts.

Keywords Perfluoroalkyl acids $\cdot$ DNA binding . Intercalation $\cdot$ Minor groove binding $\cdot$ DNA conformational change $\cdot$ DNA sequence selectivity

\section{Introduction}

Perfluoroalkyl acids (PFAAs) have been produced and used for over 50 years. Because of their specific physical and chemical properties, chemical and thermal inertness, low surface energy, and special surface-active properties, PFAAs have applications in numerous industrial and consumer products, ranging from carpet protection to firefighting foams (Lehmler 2005; Martin et al. 2004). PFAAs persist ubiquitously in the environment, resisting hydrolysis, photolysis, and biodegradation because of the high energy of the carbon-fluorine bond (Hekster et al. 2003; Martin et al. 2004; Quinete et al. 2010). Although the toxicological effects of these chemicals are still under investigation, many studies have reported on their developmental and reproductive toxicity, neurotoxicity, immunotoxicity, and carcinogenicity in animals both in vivo and in vitro (Hekster et al. 2003; Lau et al. 2004; Slotkin et al. 2008). PFAAs can cause peroxisomal proliferation, mitochondrial dysfunction, disturbance of fatty acid metabolism, hepatocellular hypertrophy, changes in 
membrane surface potential, and changes in gene expression levels (Hekster et al. 2003; Hu et al. 2002). Perfluorooctanoic acid (PFOA) and perfluorodecanoic acid are known to induce biogenesis of liver peroxisomes and significantly affect liver phospholipid metabolism (Adinehzadeh and Reo 1998). Furthermore, it has been found that perfluorinated fatty acids with a chain length of 6-10 carbons reversibly inhibits gap junctional intercellular communications (Upham et al. 1998). The down-regulation of intercellular communications by peroxisome proliferators results in abnormal cell growth and increases tumorigenicity (Trosko and Ruch 1998). Therefore, PFAAs, such as perfluorooctane sulfonate (PFOS), PFOA, and perfluorooctanesulfonyl fluoride (PFOSF), have many negative effects in the environment and have been identified as new persistent organic pollutants (Hekster et al. 2003).

To understand the molecular toxicity of these pollutants, interactions of PFAAs and several proteins have been studied. Some PFAAs bind to human serum albumin (HSA) at the same site as fatty acids with a similar affinity. The tested PFAAs have the ability to compete and displace fatty acids from the protein, and consequently change the distribution between free and bound fatty acids in plasma (Chen and Guo 2009). PFOS was demonstrated to bind strongly to bovine serum albumin (BSA) (Jones et al. 2003). Noncovalent interactions of PFOS with BSA alter its secondary conformation, and such changes inhibit the physiological function of BSA to transport vitamin $B_{2}$ (Wu et al. 2009). The non-covalent interaction between PFOA and HSA arises from hydrogen bonding, van der Waals forces, and a hydrophobic stack. The interaction of PFOA with HSA is spontaneous and results in a change in the conformation of HSA (Zhang et al. 2009b). An in vitro study has supported the hypothesis that PFAAs containing a chain of seven or eight perfluorinated carbons may interfere with the binding of fatty acids or other endogenous ligands to the liver-fatty acid binding protein (Luebker et al. 2002). Moreover, competitive binding of PFAAs to the thyroid hormone transport protein transthyretin has been investigated (Weiss et al. 2009).

Although the interactions of PFAAs with several proteins have been characterized, studies examining the interaction between PFAAs and DNA remain sparse. As mentioned above, PFAAs are known carcinogens (Rosen et al. 2009). It has long been recognized that DNA interactions with carcinogens represent an indicator of their carcinogenicity (Brookes and Lawley 1964). Therefore, the study of the quantitative binding and sequence preference between PFAA with DNA is crucial for understanding PFAA genotoxicity. Only a few studies have revealed that PFOS and PFOA can non-covalently interact with DNA and change the conformation of the DNA ( $\mathrm{Li}$ et al. 2010; Zhang et al. 2009a, b). Electrostatic attraction and hydrophobic interactions were suggested to facilitate the binding of PFOS into the grooves of DNA. The binding of PFOA to DNA corresponded to the Langmuir adsorption isotherm in a two-step binding model, in which PFOA climbed along the backbone of the DNA molecule and then interacted with the homolateral bases via hydrophobic interactions.

In our previous study, we used a rapid fluorescence displacement method in conjunction with molecular docking analysis to study the binding mode, binding affinity, and intermolecular interaction of particular organic pollutants with DNA (Wei and Guo 2009; Wei et al. 2010). Circular dichroism (CD) has been shown to conveniently detect conformational changes as well as the secondary and tertiary structural stabilities of biomacromolecules upon interaction with small chemical molecules (Ashoka et al. 2006). In the present study, we investigated the noncovalent binding interactions between five diversely structured PFAAs and seven different DNA sequences using the rapid fluorescence displacement method combined with molecular docking analysis and CD spectroscopy. The information obtained from this study should enhance our understanding of PFAAs genotoxicity.

\section{Materials and methods}

\section{Reagents}

Perfluorobutyl acid $\left(\mathrm{F}_{3} \mathrm{C}\left(\mathrm{CF}_{2}\right)_{2} \mathrm{COOH}\right.$, PFBA, purity $\left.98 \%\right)$, perfluorobutane sulfonate $\left(\mathrm{F}_{3} \mathrm{C}\left(\mathrm{CF}_{2}\right)_{3} \mathrm{SO}_{3} \mathrm{H}\right.$, PFBS, purity $97 \%)$, PFOA $\left(\mathrm{F}_{3} \mathrm{C}\left(\mathrm{CF}_{2}\right)_{6} \mathrm{COOH}\right.$, purity $\left.96 \%\right)$, PFOS $\left(\mathrm{F}_{3} \mathrm{C}\left(\mathrm{CF}_{2}\right)_{7} \mathrm{SO}_{3} \mathrm{H}\right.$, purity $\left.98 \%\right)$, perfluorododecanoic acid $\left(\mathrm{F}_{3} \mathrm{C}\left(\mathrm{CF}_{2}\right)_{11} \mathrm{COOH}\right.$, PFDoA, purity $95 \%$ ), thiazole orange (TO, purity $\sim 90 \%$ ), double-stranded calf thymus DNA (CTDNA, 13,000 base pairs), polydeoxyadenylic acid • polythymidylic acid sodium salt (poly[dA] • poly[dT]), and polydeoxyguanylic acid $\bullet$ polydeoxycytidylic acid sodium salt (poly $[\mathrm{dG}] \cdot$ poly $[\mathrm{dC}]$ ) were purchased from SigmaAldrich (St. Louis, MO, USA). Hoechst 33258 (H33258, purity $99.1 \%$ ) was purchased from Merck (Whitehouse Station, NJ, USA). Twenty base-pair double-stranded oligonucleotides - alternating copolymers deoxyadenylicthymidylic acid (oligo AT) and deoxyguanylic-cytidylic acid (oligo GC) - and homopolymers deoxyadenylic acid (oligo A) and deoxyguanylic acid (oligo G) were obtained by hybridizing two complementary single-stranded oligonucleotides purchased from Invitrogen (Shanghai, China). Every two complementary single-stranded oligonucleotides were mixed in $2 \times \mathrm{SSC}$ buffer $(0.3 \mathrm{M} \mathrm{NaCl}, 30 \mathrm{mM}$ sodium citrate, $\mathrm{pH}$ 7.0), denatured at $95{ }^{\circ} \mathrm{C}$ for $5 \mathrm{~min}$ on a Biometra TGradient thermocycler (Göttingen, Germany), and then naturally cooled to room temperature. The concentration of the oligonucleotide was determined by absorbance at $260 \mathrm{~nm}$. All other chemicals and solvents were of analytical grade. 


\section{Fluorescence measurements}

Fluorescence emission spectra were recorded on a PerkinElmer LS 55 fluorescence spectrometer at $20{ }^{\circ} \mathrm{C}$ in a right-angle configuration, a 1-cm pathlength quartz cuvette was used to assess the binding of the fluorescent probes to DNA, a 3$\mathrm{mm}$ pathlength quartz cuvette was used to assess the binding of PFAAs to DNA by fluorescence displacement measurements, and a 10-nm slit-width was set for excitation and emission. The excitation wavelengths were 504 and $344 \mathrm{~nm}$ for TO and H33258, respectively. The emission spectra were recorded between 510 and $560 \mathrm{~nm}$ for TO, and between 400 and $520 \mathrm{~nm}$ for H33258. The DNA was dissolved in $50 \mathrm{mM}$ Tris-HCl buffer ( $\mathrm{pH}$ 7.4). Stock solutions of the tested organic compounds were prepared in methanol and then added to the dye/DNA solution with a final methanol content of $<5 \%$.

\section{Data analysis}

To determine the dissociation constants of the fluorescent dye with DNA, the intensity values corresponding to the maximum fluorescence emission were plotted against the dye to base-pair ratios. The curves were linearized by Scatchard analysis (Boger et al. 2001). The dye was mixed with DNA at a stoichiometric ratio and the PFAAs were determined from the fluorescence intensity values. The dissociation constants of the PFAAs were calculated from the inhibitory concentration at half fluorescence intensity [ $\left.\mathrm{IC}_{50}\right]$ using the equation $K_{\mathrm{d}}=\left[\mathrm{IC}_{50}\right] /\left(1+[\right.$ dye $\left.] / K_{\text {dye }}\right)$, where $K_{\mathrm{d}}$ is the dissociation constant, [dye] is the concentration of the fluorescence dye, and $K_{\text {dye }}$ is the dissociation constant of the dye with the nucleic acid (Cheng and Prusoff 1973).

\section{Circular dichroism (CD) measurements}

CD measurements were performed on a JASCO J-815 spectropolarimeter (Tokyo, Japan) with a 1-mm pathlength quartz cuvette. The CD spectra were recorded from 185 to $400 \mathrm{~nm}$ at a scan speed of $50 \mathrm{~nm} / \mathrm{min}$ and a 1 -s response time. In experiments where a PFAA prepared in methanol was titrated into a CT-DNA solution in $50 \mathrm{mM}$ Tris- $\mathrm{HCl}$ buffer ( $\mathrm{pH} 7.4$ ), the final amount of methanol was below $5 \%$ to minimize potential interference.

\section{Molecular docking analysis}

Three ligand-DNA structures used for molecular docking were acquired from the Protein Data Bank (PDB) (http:// www.rcsb.org/pdb) with PDB identification numbers $1 \mathrm{Z} 3 \mathrm{~F}$, 108D, and 296D. The three-dimensional structure building of small molecular ligands and all modeling were performed using the molecular modeling software package Sybyl 7.3
(Tripos Inc., St. Louis, MO, USA) running on a Linux workstation. Tripos standard molecular field, GasteigerHückel charge, and the Powell energy optimization strategy were used for the energy minimizations and optimization of ligand structures. The energy convergence criterion was fixed at $0.05 \mathrm{kcal} / \mathrm{mol}$, maximum iterations times were set at 1,000 , the non-bond cutoff of $8 \AA$ was adopted to consider intramolecular interactions, and other parameters were used as default values. The binding interactions between the PFAAs and nucleic acid molecules were analyzed by the Surflex-dock module in Sybyl 7.3. Surflex-dock used an empirical scoring function and a patented search engine to dock ligands into a binding site. The scoring function of Surflex-dock included hydrophobic, polar, repulsive, entropic, and solvation terms.

\section{Results and discussion}

Binding studies of fluorescent probes to double-stranded oligonucleotides

Similar to our previous investigation examining the affinity of the fluorescent probes to CT-DNA, poly[dA] $\bullet$ poly[dT] and poly $[\mathrm{dG}] \cdot \operatorname{poly}[\mathrm{dC}]$, the binding interaction of $\mathrm{TO}$ and H33258 with the four double-stranded oligonucleotides was characterized herein (Wei and Guo 2009). The $K_{\mathrm{d}}$ values of TO with the oligonucleotides were calculated to be 0.17 , $0.18,0.40$, and $0.18 \mu \mathrm{M}$ for oligo AT, oligo GC, oligo A, and oligo $\mathrm{G}$, respectively. The binding stoichiometry of all four oligonucleotides was 1.5 DNA base pairs for each TO molecule. The $K_{\mathrm{d}}$ values of $\mathrm{H} 33258$ with oligo AT and oligo A were determined to be 14 and $2.8 \mathrm{nM}$, respectively. The binding stoichiometry of both oligonucleotides was 5 bp for each H33258 molecule. In contrast, there was essentially no fluorescence of $\mathrm{H} 33258$ when in the presence of the oligo GC or oligo G samples, as observed previously for poly $[\mathrm{dG}] \cdot$ poly $[\mathrm{dC}]$. Therefore, the binding preference of $\mathrm{H} 33258$ towards AT sequences was observed. The binding stoichiometry and dissociation constants were determined in the titration experiment with the probes added to a CT-DNA solution, and were 1.5 and $0.14 \mu \mathrm{M}$, respectively, for TO, and 20 and $3 \mathrm{nM}$, respectively, for H33258 (Wei and Guo 2009). The TO binding stoichiometry and dissociation constant were determined to be 2.3 and $0.05 \mu \mathrm{M}$ with poly[dA] • poly[dT], and 1.4 and $0.18 \mu \mathrm{M}$ with poly[dG] • poly[dC] (Wei and Guo 2009). A comparison of the results shows that the binding of TO to oligo A was 8-fold weaker than binding to poly $[\mathrm{dA}] \cdot$ poly $[\mathrm{dT}]$, whereas binding of TO to oligo A was 2-fold weaker than binding to the other three oligonucleotides. Moreover, oligo AT and oligo A bound more H33258 molecules than CT-DNA. Oligo A showed similar H33258 affinity when compared with the results for 
CT-DNA, and oligo AT showed 5-fold weaker H33258 affinity than oligo A and CT-DNA.

\section{Binding of perfluoroalkyl acids with DNA}

After studying the binding affinities of fluorescent probes to DNA, the binding of the five PFAAs (PFOS, PFOA, PFBA, PFBS, and PFDoA) with DNA was investigated by the fluorescence displacement method. In the measurements, the intercalative or minor groove binding probe was treated as a reporter and mixed with the DNA at a stoichiometric ratio, and PFAAs were treated as competitors.

In competitive binding experiments with the intercalative probe TO, the five PFAAs bound to seven kinds of DNA with different affinities. For all the PFAAs, the fluorescence signal decreased to near background levels because of their good solubility in aqueous solutions. The displacement curves are shown in Fig. 1 and S1, and the calculated $K_{\mathrm{d}}$ values describing the affinities of these PFAAs to the seven different DNA molecules are listed in Table 1 . The $K_{\mathrm{d}}$ values for the examined PFAAs ranged from 0.11 to $1,217.14 \mu \mathrm{M}$. The values for PFDoA were the lowest; thus, it bound to DNA more strongly than the other PFAAs. In contrast, PFBA showed the weakest binding affinity. The strongest to weakest binding affinity order of the five PFAAs was PFDoA, PFOS, PFOA, PFBS, and PFBA.

For either perfluoroalkyl sulfonates (PFOS and PFBS) or perfluorocarboxyl acids (PFBA, PFOA, and PFDoA), the compounds with longer carbon chains had stronger DNA binding affinities. PFOS contains an eight-carbon chain and had stronger DNA binding affinity than PFBS with a fourcarbon chain. Similarly, PFDoA, PFOA, and PFBA with 13, eight, and four carbon chains, respectively, showed decreasing DNA binding affinities. A comparison of the perfluoroalkyl sulfonates and perfluorocarboxyl acids with

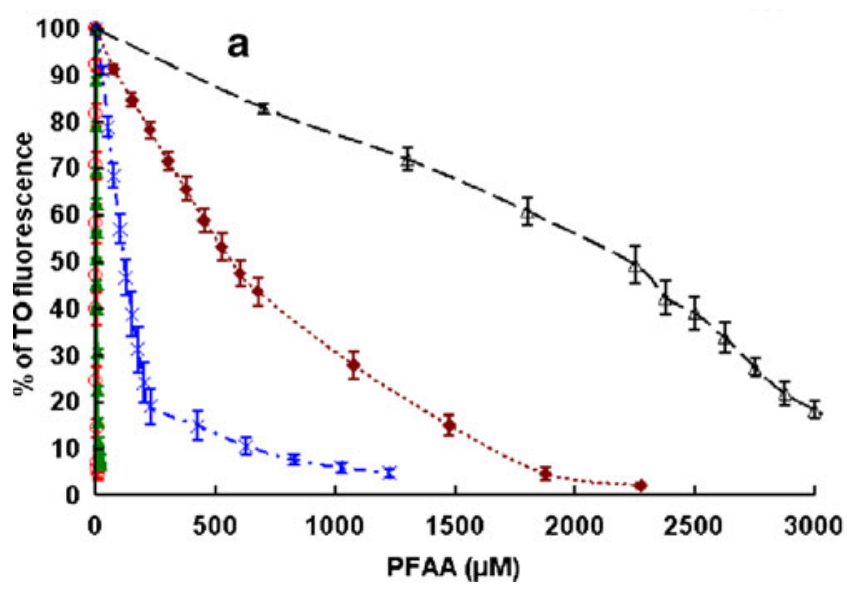

Fig. 1 Fluorescence displacement curves of PFAAs: PFOS (filled triangles), PFOA (asterisks), PFDoA (open circles), PFBS (filled diamonds), and PFBA (open triangles), which were titrated into the same number of carbons showed that PFOS and PFBS had stronger binding affinities than PFOA and PFBA, respectively. Hydrogen bonds have been proposed to form between the fluorine and oxygen atoms of the ligand and DNA upon interaction (Wei et al. 2010). Consequently, as there are more donor fluorine and oxygen atoms on the perfluoroalkyl sulfonates than on the perfluorocarboxyl acids with same number of carbons, it is likely that more hydrogen bonds form, thereby accounting for the observed stronger affinity of PFOS and PFBS to DNA than PFOA and PFBA. Similarly, perfluoroalkyl sulfonate or perfluorocarboxyl acid with longer carbon chains have more fluorine atoms and bind to DNA more strongly.

For each studied PFAA, different affinities towards the various sequences were observed. The data showed that besides PFBA, the other PFAAs showed the highest affinity towards CT-DNA. Among the four oligonucleotides, with the exception of PFBS, the other PFAAs showed the strongest binding affinity to oligo AT. In addition, excluding PFOS, the other PFAAs bound the weakest to oligo A. Different binding affinities of the PFAAs towards poly[dA] poly $[\mathrm{dT}]$ and poly $[\mathrm{dG}] \cdot$ poly $[\mathrm{dC}]$ were observed. PFOS and PFOA preferentially bound to poly $[\mathrm{dG}] \cdot \operatorname{poly}[\mathrm{dC}]$, whereas PFBS and PFBA preferentially bound to poly[dA] $\cdot$ poly[dT]. PFDoA showed the same binding affinity to both poly[dA] poly $[\mathrm{dT}]$ and poly $[\mathrm{dG}] \cdot$ poly $[\mathrm{dC}]$. Comparing the binding affinities of PFAAs with oligonucleotides and a long-chain DNA duplex, the PFAAs exhibited stronger binding affinity to oligo AT than towards poly[dA] $\cdot$ poly[dT] or oligo A. The PFAAs showed different binding preferences toward poly $[\mathrm{dG}] \cdot$ poly $[\mathrm{dC}]$, oligo $\mathrm{GC}$, and oligo $\mathrm{G}$.

Moreover, competitive binding experiments for the PFAAs were performed using the minor groove binding agent $\mathrm{H} 33258$ as the probe. At the excitation wavelength for H33258, essentially no fluorescence arising from

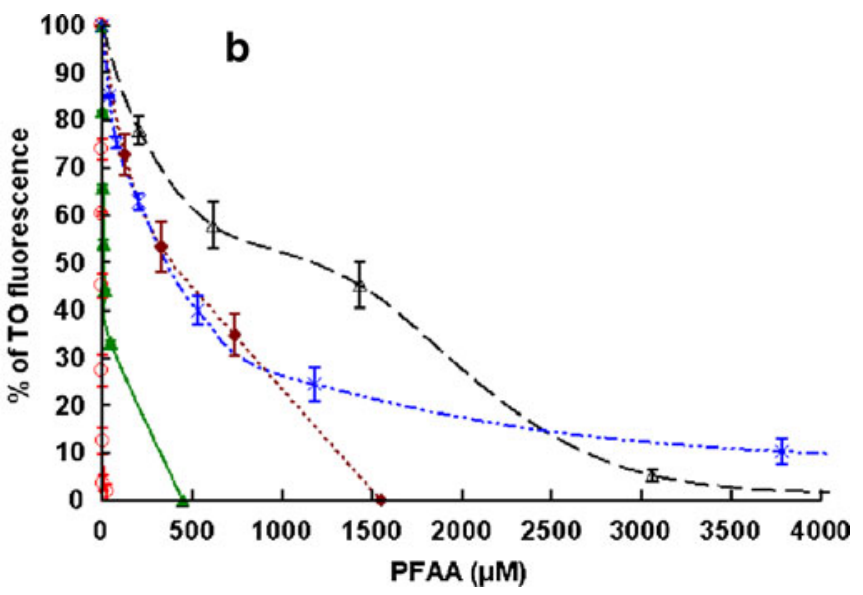

$1.0 \mu \mathrm{g} / \mathrm{mL}$ of CT-DNA $+1.0 \mu \mathrm{M}$ of $\mathrm{TO}(\mathbf{a})$, or $0.5 \mu \mathrm{g} / \mathrm{mL}$ of oligo $\mathrm{GC}+0.5 \mu \mathrm{M}$ of $\mathrm{TO}(\mathbf{b})$. Each concentration was repeated in triplicate 
Table 1 Dissociation constants $\left(K_{\mathrm{d}}\right)$ of PFAAs with oligonucleotides, poly $[\mathrm{dA}] \cdot \operatorname{poly}[\mathrm{dT}]$, poly $[\mathrm{dG}] \cdot \operatorname{poly}[\mathrm{dC}]$, and CTDNA obtained by fluorescence displacement measurements with $\mathrm{TO}$ and $\mathrm{H} 33258$ as probes

\begin{tabular}{|c|c|c|c|c|c|c|}
\hline \multirow[t]{2}{*}{ Probe } & \multirow[t]{2}{*}{ DNA } & \multicolumn{5}{|c|}{$K_{\mathrm{d}}(\mu \mathrm{M})$} \\
\hline & & PFOS & PFOA & PFDoA & PFBS & PFBA \\
\hline \multirow[t]{7}{*}{$\mathrm{TO}$} & CT-DNA & 0.37 & 13.51 & 0.11 & 66.23 & 289.86 \\
\hline & Oligo AT & 1.11 & 24.00 & 0.23 & 229.28 & 260.22 \\
\hline & Oligo A & 9.14 & 98.86 & 0.60 & 614.29 & $1,217.14$ \\
\hline & Poly $[\mathrm{dA}] \cdot \operatorname{poly}[\mathrm{dT}]$ & 17.00 & 282.50 & 0.44 & 243.75 & 335.00 \\
\hline & Oligo GC & 2.93 & 94.93 & 0.29 & 103.50 & 312.00 \\
\hline & Oligo G & 12.85 & 50.81 & 0.24 & 275.71 & 546.95 \\
\hline & Poly $[\mathrm{dG}] \cdot \operatorname{poly}[\mathrm{dC}]$ & 3.78 & 96.00 & 0.44 & 278.27 & 653.56 \\
\hline \multirow[t]{7}{*}{ H33258 } & CT-DNA & 45.45 & 172.41 & 30.30 & 141.64 & 226.76 \\
\hline & Oligo AT & 207.66 & 2141.21 & 3.46 & - & - \\
\hline & Oligo A & 382.98 & 1117.73 & 10.78 & - & - \\
\hline & Poly $[\mathrm{dA}] \cdot \operatorname{poly}[\mathrm{dT}]$ & - & - & - & - & - \\
\hline & Oligo GC & - & - & - & - & - \\
\hline & Oligo G & - & - & - & - & - \\
\hline & $\operatorname{Poly}[\mathrm{dG}] \cdot \operatorname{poly}[\mathrm{dC}]$ & - & - & - & - & - \\
\hline
\end{tabular}

H33258 in the oligo GC, oligo G, and poly $[\mathrm{dG}] \cdot \operatorname{poly}[\mathrm{dC}]$ samples was observed. However, in the poly[dA] $\operatorname{poly}[\mathrm{dT}]$ duplex, the signal increased steadily in a non-saturating manner over the range of concentrations studied (Wei and Guo 2009). Therefore, the PFAAs could be tested using the displacement experiment with oligo AT, oligo A, and CTDNA (Fig. 2 and S2). The $K_{\mathrm{d}}$ values for the PFAAs with the three kinds of DNA molecules ranged from 3.46 to 2,141.21 $\mu \mathrm{M}$ (Table 1). PFBS or PFBA in high concentrations shifted the fluorescence emission peak of H33258 in oligo AT and oligo A samples. Therefore, the $K_{\mathrm{d}}$ values of PFBS and PFBA with oligo AT and oligo A could not be obtained. The obtained $K_{\mathrm{d}}$ values showed that PFDoA bound to DNA more strongly than the other PFAAs, and the binding affinities of PFDoA, PFOS, and PFOA

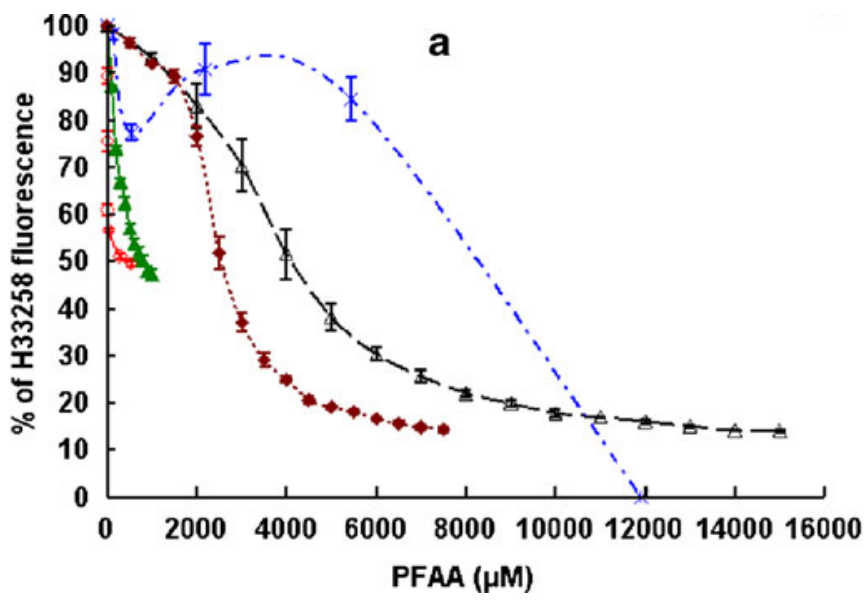

Fig. 2 Fluorescence displacement curves of PFAAs: PFOS (filled triangles), PFOA (asterisks), PFDoA (open circles), PFBS (filled diamonds), and PFBA (open triangles), which were titrated into decreased in that order. A similar trend for those three PFAAs was observed for results examining DNA intercalation of these compounds. Moreover, besides PFBA binding to CT-DNA, the other PFAAs showed much weaker minor groove binding affinities than their intercalative binding with DNA. Although the limited number of PFAAs examined precludes any firm conclusions to be drawn, the results indicate that PFAAs with longer carbon chains with more fluorine atoms should have a stronger minor groove binding affinity. Moreover, perfluoroalkyl sulfonate of a particular carbon length appears to have a stronger minor groove binding affinity than the corresponding perfluorocarboxyl acid.

The binding of PFAAs with DNA was different to organic pollutants with plane formations, i.e., polycyclic aromatic

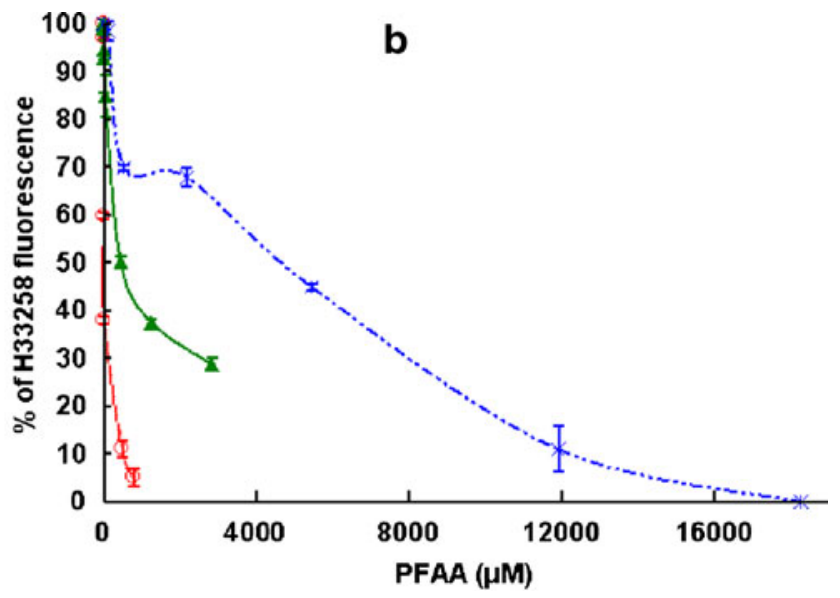

$0.5 \mu \mathrm{g} / \mathrm{mL}$ of CT-DNA $+0.05 \mu \mathrm{M}$ of $\mathrm{H} 33258$ (a), or $0.2 \mu \mathrm{g} / \mathrm{mL}$ of oligo AT $+0.06 \mu \mathrm{M}$ of $\mathrm{H} 33258$ (b). Each concentration was repeated in triplicate 
hydrocarbons (PAHs) (Wei and Guo 2009). Although both PFAAs and PAHs preferentially intercalate into DNA rather than bind to grooves, the tested PFAAs did bind into the minor groove with varying affinities. Intercalation of PFOS and groove binding of PFOA with DNA were reported previously (Li et al. 2010; Zhang et al. 2009a). In contrast, no groove binding was observed for PAHs. We assumed that the molecular shape of ligands affects their DNA binding mode. The molecular plane structures of ligands appear to favor intercalation and preclude groove binding.

Conformational changes to DNA upon interaction with PFAAs

The covalent and non-covalent interactions in biological macromolecules such as DNA could conduce to forming specific conformations. When pollutants or toxicants interact with DNA, the internal non-covalent bonds of a DNA duplex molecule are often disrupted, and such events possibly alter the conformation of the DNA or the specific function of the DNA molecule (Lah et al. 2008). CD spectroscopy is a powerful technique to monitor DNA conformational changes resulting from ligand binding events (Chang et al. 2012). B-form DNA is characterized by a positive long wavelength band at about $260-280 \mathrm{~nm}$ due to base stacking and a negative band around $245 \mathrm{~nm}$ due to helicity. In addition, the position and amplitude of the CD bands show marked differences because of sequence diversity (Alexeev et al. 1987; Nelson et al. 1987; Trantirek et al. 2000).

In this study, the conformational changes of CT-DNA binding to TO or H33258 were investigated initially. As shown in Fig. S3, the CD spectra of CT-DNA in the 225-305 nm range indicated the presence of a B-form helix with a negative band at $246 \mathrm{~nm}$ and a positive band at $275 \mathrm{~nm}$. Decreases in the amplitude of the CD signal at both 246 and $275 \mathrm{~nm}$ in the presence of the two probes were observed. These results indicated that TO and H33258 weaken DNA base stacking and loosen DNA helicity (Tong et al. 2010). Therefore, the two probes caused DNA conformational changes. Conformational changes to the CTDNA on mixing with the five PFAAs were then examined. The results presented in Fig. 3 and S3 showed that the addition of increasing concentrations of particular PFAAs to the CT-DNA led to reductions in the amplitude of the signals at 246 and $275 \mathrm{~nm}$. The CD spectra of CT-DNA binding with different PFAAs showed that the largest changes were observed when CT-DNA was combined with PFBA and PFBS. The data indicated that these two pollutants caused larger DNA conformational changes by weakening base stacking and loosening DNA helicity when compared with the results for PFDoA, PFOS, and PFOA. Different to the fluorescence displacement data, we observed that PFAAs containing shorter carbon chain lengths give rise to larger DNA conformational changes. Zhang et al. studied conformational changes to DNA upon binding PFOA (Zhang et al. 2009a). An enhancement in the CD signal at both 245 and $275 \mathrm{~nm}$ in the presence of PFOA was observed. Nonetheless, the observed decreases in peak intensities obtained in our $\mathrm{CD}$ experiments can likely be attributed to the use of much higher PFOA concentrations and different buffer solutions.

Molecular docking analysis of non-covalent interaction of PFAAs with DNA

Although the binding models describing the interaction of the PFAAs with our tested DNA could not be obtained because the structures of related complexes were not available in the PDB, the results of the molecular docking analysis still provided useful information regarding the PFAA-DNA noncovalent interactions. The PDB contains extensive information regarding the structures of ligand-DNA complexes, and three structures of these complexes were used to establish the PFAA-DNA intercalation or the minor groove binding model to explore the binding interactions. Ellipticine intercalated into the sequence d(CGATCG) $)_{2}(1 \mathrm{Z3F})$ was selected because this structure had been used to successfully explore the intercalation of pollutants with DNA (Wei et al. 2010). The second intercalation model (108D), d(CGCTAGCG) $)_{2}$ complexed with the bis-intercalator $1,1^{\prime}$-(4,4,8,8-tetramethyl-4,8diazaundecamethylene)bis[4-(3-methyl-2,3-dihydrobenzo1,3-thiazolyl-2-methylidene)quinolinium] tetraiodide (TOTO), was selected because the binding mode of TOTO is similar to that of TO (Hansen et al. 1996). H33258 binding to d(CGCAAATTTGCG) $)_{2}$ (296D) was used as the minor groove binding model.

The molecular docking scores for the interactions between the tested PFAAs and the three oligonucleotide models are summarized in Table 2. The score values ranged from 1.91 to 4.31, from 1.43 to 4.13 , and from 2.75 to 4.85 for $\mathrm{d}$ $(\text { CGATCG })_{2}$, d(CGCTAGCG $)_{2}$, and d(CGCAAATTTGCG $)_{2}$, respectively. For each model, a large score indicated stronger binding affinity of the small molecules to the DNA. Therefore, PFAAs with larger score values should have smaller $K_{\mathrm{d}}$ values. As expected, PFDoA had the highest docking score values and this corresponds with this molecule having the highest groovebinding affinity or strongest affinity to intercalate into the DNA. Significant correlations were revealed between docking score and log-normalized $K_{\mathrm{d}}$ values for all of the tested PFAAs (Fig. 4). The $R^{2}$ values were $0.6449,0.6079$, and 0.8384 for intercalation $\mathrm{p} K_{\mathrm{d}}$ to Score $_{1 \mathrm{Z3F}}$, intercalation $\mathrm{p} K_{\mathrm{d}}$ to Score $_{108 \mathrm{D}}$ and minor groove binding $\mathrm{p} K_{\mathrm{d}}$ to $\mathrm{Score}_{296 \mathrm{D}}$, respectively. The significant negative relationship also confirmed that the tested PFAAs interacted with the DNA by both intercalation and minor groove binding modes. 

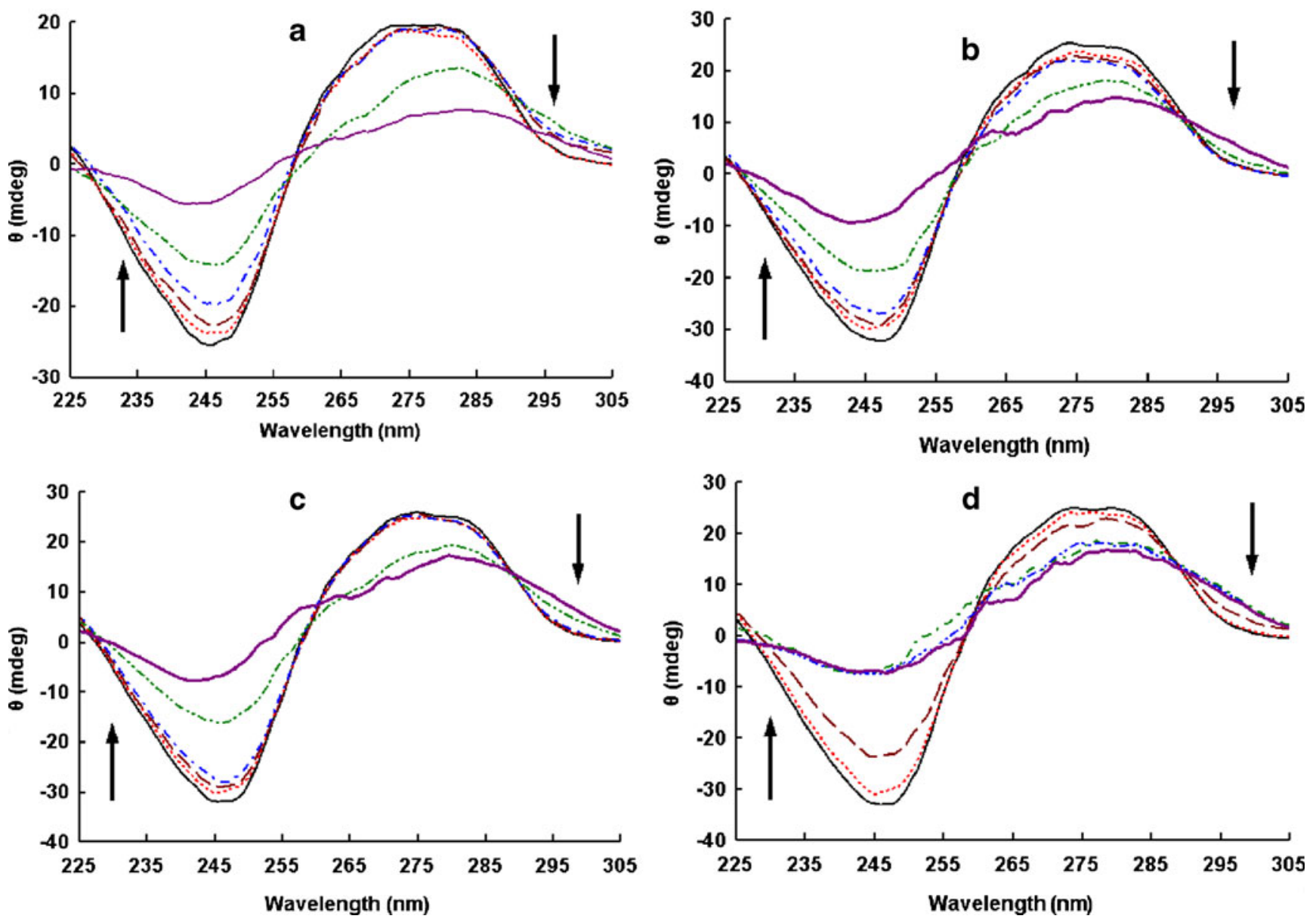

Fig. 3 CD spectra of 0, 7.7, 14.8, 21.4, 27.6, and 33.3 mM of PFDoA (a) or PFOA (b); 0, 1.0, 2.0, 2.9, 3.8, and 4.8 mM of PFBS (c); or 0, 2.0, 3.8, $5.7,7.4$, and $9.1 \mathrm{mM}$ of PFBA (d) titrated into $0.8 \mathrm{mg} / \mathrm{mL}$ of CT-DNA. The arrows indicate the increasing PFAA concentration for each line

Toxicity evaluation

As mentioned in the "Introduction", humans have been exposed to a large quantity of perfluorinated compounds. The quantitatively dominant component of PFAAs in human blood is PFOS whose concentrations are in the range of 3 to $55 \mu \mathrm{g} / \mathrm{L}$ (Stahl et al. 2011; Vierke et al. 2012). The PFOA concentrations, in the range of 1 to $12 \mu \mathrm{g} / \mathrm{L}$, are generally somewhat lower in the blood than PFOS concentrations. The blood concentrations of other PFAAs, such as PFBS, PFBA, and PFDoA, are one or two orders of magnitude lower than that of PFOA. Moreover, since PFOS and PFOA cannot be metabolized by mammals, excretion is the only means by which the toxic activity of these compounds can be eliminated once they have been taken up by the body. According to current knowledge, PFOS is excreted more slowly than PFOA so that the latter has a shorter elimination half-life and higher rate of excretion. Short-chain PFAAs such as PFBS and PFBA are excreted more rapidly than long-chain PFAAs.

Our study shows that long-chain PFAAs have stronger DNA binding affinities than short-chain PFAAs, such as

Table 2 Log-normalized $K_{\mathrm{d}}$ values $\left(\mathrm{p} K_{\mathrm{d}}\right.$ ) for PFAAs binding to CT-DNA, and molecular docking scores of the intercalation of PFAAs into d $(\mathrm{CGATCG})_{2}\left(\right.$ Score $\left._{1 \mathrm{Z3}}\right), \mathrm{d}(\mathrm{CGCTAGCG})_{2}\left(\right.$ Score $\left._{108 \mathrm{D}}\right)$, and d(CGCAAATTTGCG $)_{2}\left(\right.$ (core $\left._{296 \mathrm{D}}\right)$ base stacking

\begin{tabular}{llllll}
\hline PFAAs & & PFOS & PFOA & PFDoA & PFBS \\
\hline Intercalation & $\mathrm{p} K_{\mathrm{d}}$ & 6.432 & 4.869 & 6.959 & 4.179 \\
& Score $_{1 \mathrm{Z3F}}$ & 3.55 & 3.27 & 4.31 & 3.538 \\
& Score $_{108 \mathrm{D}}$ & 2.29 & 2.94 & 4.13 & 2.93 \\
Minor groove binding & $\mathrm{p} K_{\mathrm{d}}$ & 4.342 & 3.763 & 4.519 & 1.43 \\
& $\mathrm{Score}_{296 \mathrm{D}}$ & 4.36 & 3.57 & 4.85 & 3.849 \\
& & & & 2.75 \\
\hline
\end{tabular}




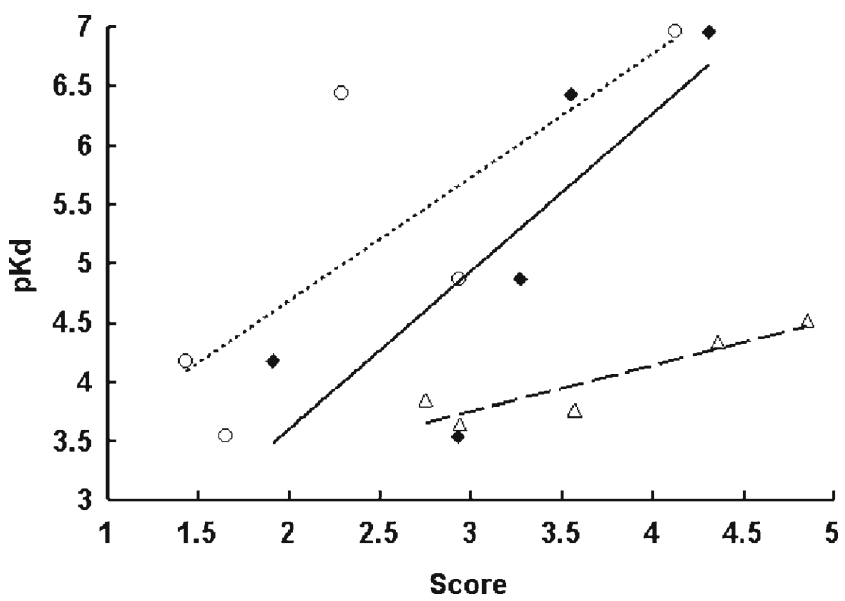

Fig. 4 Correlations between the molecular docking scores of the PFAAs intercalating into d(CGATCG) $)_{2}$ (filled diamonds) and $\mathrm{d}$ $(\mathrm{CGCTAGCG})_{2}$ (open circles), and the log-normalized $K_{\mathrm{d}}$ values $\left(\mathrm{p} K_{\mathrm{d}}\right)$ of the PFAAs intercalating into CT-DNA. Correlations between the molecular docking scores of the PFAAs minor groove binding to the oligonucleotide d(CGCAAATTTGCG) ${ }_{2}$ (open triangles) and the $\mathrm{p} K_{\mathrm{d}}$ values of the PFAAs minor groove binding to CT-DNA

PFBS and PFBA. Therefore, we can assume that the longchain PFAAs are more toxic than the short-chain PFAAs which are more rapidly excreted. Among the five studied PFAAs, PFDoA exhibits the highest DNA affinity. However, PFDoA has lower concentrations in the general environment or in human blood than PFOS and PFOA. Thus, we can expect that PFDoA still exhibits non-significant toxic effects. Obviously, PFOS is the most harmful compound among the five studied pollutants because of the high blood concentration, slow excretion, and strong DNA binding affinity. However, if human exposure to PFDoA increased, PFDoA would be more harmful than the other studied PFAAs. Finally, based on the toxicity evaluation, we suggest to decrease PFOS and long-chain PFAAs production due to environmental protection and human health effects.

\section{Conclusions}

In the present study, we investigated the binding mode and binding affinity of five PFAAs to seven types of DNA using fluorescence displacement and molecular docking analysis. Moreover, DNA conformational changes upon binding of PFAAs were also reported by CD measurements. The data revealed that these PFAAs predominantly interacted with DNA by intercalation; however, the PFAAs also showed groove binding affinity. In the competitive binding experiments where TO was the probe, the strongest to the weakest binding affinity order of the five PFAAs was PFDoA, PFOS, PFOA, PFBS, and PFBA. The $K_{\mathrm{d}}$ values for the examined PFAAs ranged from 0.11 to $1,217.14 \mu \mathrm{M}$. The compounds with longer carbon chains showed stronger
DNA binding affinities. By comparing the perfluoroalkyl sulfonates and perfluorocarboxyl acids that contain the same number of carbons, PFOS and PFBS showed stronger binding affinities than PFOA and PFBA, respectively. Presumably the higher content of fluorine and oxygen atoms within a PFAA could provide more hydrogen bond donors and thus facilitate stronger DNA intercalation. Except PFBA, the PFAAs bound more strongly to CT-DNA than to the other six types of DNA duplexes tested. Among the four oligonucleotides, the PFAAs showed the strongest binding affinity towards oligo AT (except PFBS). When the DNA groove binding agent $\mathrm{H} 33258$ was used as the probe, PFDoA bound to DNA more strongly than the other PFAAs, as observed for the intercalative binding affinity assay. The $K_{\mathrm{d}}$ values for PFAAs with the oligo AT, oligo A, and CT-DNA ranged from 3.46 to $2,141.21 \mu \mathrm{M}$. Moreover, except for the binding of PFBA to CT-DNA, the other PFAAs showed much weaker minor groove binding affinities when compared with their corresponding intercalative binding affinities. The minor groove binding affinities of PFOS, PFOA, and PFDoA followed the same trend as observed for the intercalation data. The DNA binding mode and affinities of the PFAAs were also confirmed by molecular docking analysis because significant correlations were revealed between the docking scores and the lognormalized $K_{\mathrm{d}}$ values for all of the tested PFAAs. Moreover, DNA conformational changes upon binding of PFAAs were examined. The five PFAAs weakened DNA base stacking and also loosened DNA helicity. The carcinogenesis of PFAAs should be investigated further by examining the covalent and non-covalent binding interactions between more PFAAs and different genes. This study is now underway in our laboratory. We also assumed that the long-chain PFAAs were more toxic than the short-chain PFAAs which were more rapidly excreted. PFOS was the most harmful compound among the five studied pollutants. Reduction of PFOS and long-chain PFAAs production was suggested.

Acknowledgments This study was supported by the National Natural Science Foundation of China (20907068 and 21077124), the Science and Technology Program of the General Administration of Quality Supervision, Inspection and Quarantine of the P.R.C. (2012IK219), and the National Natural Science Foundation of Fujian Province (2011J05015).

\section{References}

Adinehzadeh M, Reo NV (1998) Effects of peroxisome proliferators on rat liver phospholipids: sphingomyelin degradation may be involved in hepatotoxic mechanism of perfluorodecanoic acid. Chem Res Toxicol 11:428-440

Alexeev DG, Lipanov AA, Skuratovskii I (1987) Poly(dA) poly(dT) is a B-type double helix with a distinctively narrow minor groove. Nature 325:821-823 
Ashoka S, Seetharamappa J, Kandagal PB, Shaikh SMT (2006) Investigation of the interaction between trazodone hydrochloride and bovine serum albumin. J Lumin 121:179-186

Boger DL, Fink BE, Brunette SR, Tse WC, Hedrick MP (2001) A simple, high-resolution method for establishing DNA binding affinity and sequence selectivity. J Am Chem Soc 123:5878-5891

Brookes P, Lawley PD (1964) Evidence for the binding of polynuclear aromatic hydrocarbons to the nucleic acids of mouse skin: relation between carcinogenic power of hydrocarbons and their binding to deoxyribonucleic acid. Nature 202:781-784

Chang YM, Chen CKM, Hou MH (2012) Conformational changes in DNA upon ligand binding monitored by circular dichroism. Int J Mol Sci 13:3394-3413

Chen YM, Guo LH (2009) Fluorescence study on site-specific binding of perfluoroalkyl acids to human serum albumin. Arch Toxicol 83:255-261

Cheng YC, Prusoff WH (1973) Relationship between the inhibition constant $\left(\mathrm{K}_{\mathrm{I}}\right)$ and the concentration of inhibitor which causes 50 per cent inhibition $\left(\mathrm{I}_{50}\right)$ of an enzymatic reaction. Biochem Pharmacol 22:3099-3108

Hansen LF, Jensen LK, Jacobsen JP (1996) Bis-intercalation of a homodimeric thiazole orange dye in DNA in symmetrical pyrimidine-pyrimidine-purine-purine oligonucleotides. Nucleic Acids Res 24:859-867

Hekster FM, Laane RW, de Voogt P (2003) Environmental and toxicity effects of perfluoroalkylated substances. Rev Environ Contam Toxicol 179:99-121

Hu W, Jones PD, Upham BL, Trosko JE, Lau C, Giesy JP (2002) Inhibition of gap junctional intercellular communication by perfluorinated compounds in rat liver and dolphin kidney epithelial cell lines in vitro and Sprague-Dawley rats in vivo. Toxicol Sci 68:429-436

Jones PD, Hu W, De Coen W, Newsted JL, Giesy JP (2003) Binding of perfluorinated fatty acids to serum proteins. Environ Toxicol Chem 22:2639-2649

Lah J, Drobnak I, Dolinar M, Vesnaver G (2008) What drives the binding of minor groove-directed ligands to DNA hairpins? Nucleic Acids Res 36:897-904

Lau C, Butenhoff JL, Rogers JM (2004) The developmental toxicity of perfluoroalkyl acids and their derivatives. Toxicol Appl Pharm 198:231-241

Lehmler HJ (2005) Synthesis of environmentally relevant fluorinated surfactants - a review. Chemosphere 58:1471-1496

Li L, Pan Q, Xu ZS, Song GW (2010) Resonance light scattering method for study on the interaction between fluorinated surfactant potassium perfluorooctanesulfonate and DNA. J Disper Sci Technol 31:1691-1696

Luebker DJ, Hansen KJ, Bass NM, Butenhoff JL, Seacat AM (2002) Interactions of flurochemicals with rat liver fatty acid-binding protein. Toxicology 176:175-185

Martin JW, Smithwick MM, Braune BM, Hoekstra PF, Muir DCG, Mabury SA (2004) Identification of long-chain perfluorinated acids in biota from the Canadian Arctic. Environ Sci Technol 38:373-380

Nelson HC, Finch JT, Luisi BF, Klug A (1987) The structure of an oligo(dA) - oligo(dT) tract and its biological implications. Nature 330:221-226

Quinete N, Orata F, Maes A, Gehron M, Bauer KH, Moreira I, Wilken RD (2010) Degradation studies of new substitutes for perfluorinated surfactants. Arch Environ Contam Toxicol 59:20 30

Rosen MB, Lau C, Corton JC (2009) Does exposure to perfluoroalkyl acids present a risk to human health? Toxicol Sci 111:1-3

Slotkin TA, Mackillop EA, Melnick RL, Thayer KA, Seidler FJ (2008) Developmental neurotoxicity of perfluorinated chemicals modeled in vitro. Environ Health Persp 116:716-722

Stahl T, Mattern D, Brunn H (2011) Toxicology of perfluorinated compounds. Environmental Sciences Europe 23:1-52

Tong C, Hu Z, Liu W (2010) Study on the interaction between paraquat and calf thymus DNA by spectral methods. Acta Chim Sinica 68:1404-1410 (in Chinese)

Trantirek L, Stefl R, Vorlickova M, Koca J, Sklenar V, Kypr J (2000) An A-type double helix of DNA having B-type puckering of the deoxyribose rings. J Mol Biol 297:907-922

Trosko JE, Ruch RJ (1998) Cell-cell communication in carcinogenesis. Front Biosci 3:208-236

Upham BL, Deocampo ND, Wurl B, Trosko JE (1998) Inhibition of gap junctional intercellular communication by perfluorinated fatty acids is dependent on the chain length of the fluorinated tail. Int $\mathrm{J}$ Cancer 78:491-495

Vierke L, Staude C, Biegel-Engler A, Drost W, Schulte C (2012) Perfluorooctanoic acid (PFOA) - main concerns and regulatory developments in Europe from an environmental point of view. Environmental Sciences Europe 24:1-11

Wei Y, Guo LH (2009) Binding interaction between polycyclic aromatic compounds and DNA by fluorescence displacement method. Environ Toxicol Chem 28:940-945

Wei Y, Lin Y, Zhang AQ, Guo LH, Cao J (2010) Evaluation of the noncovalent binding interactions between polycyclic aromatic hydrocarbon metabolites and human p53 cDNA. Sci Total Environ 408:6285-6290

Weiss JM, Andersson PL, Lamoree MH, Leonards PEG, van Leeuwen SPJ, Hamers T (2009) Competitive binding of poly- and perfluorinated compounds to the thyroid hormone transport protein transthyretin. Toxicol Sci 109:206-216

Wu LL, Gao HW, Gao NY, Chen FF, Chen L (2009) Interaction of perfluorooctanoic acid with human serum albumin. BMC Struct Biol 9:31

Zhang X, Shen R, Chen L (2009a) Non-covalent interaction of perfluorooctanoic acid with DNA. Chinese J Chem 27:22672272

Zhang X, Chen L, Fei XC, Ma YS, Gao HW (2009b) Binding of PFOS to serum albumin and DNA: insight into the molecular toxicity of perfluorochemicals. BMC Mol Biol 10:16 